\title{
ON EIGENVALUES OF SUMS OF NORMAL MATRICES
}

\author{
HeLmut WiELANDT
}

1. Problem, notations, results. A well-known theorem due essentially to Bendixson [1, Theorem II] states that if $X$ and $Y$ are hermitian $n \times n$ matrices with eigenvalues

$$
\xi_{1} \leq \xi_{2} \leq \cdots \leq \xi_{n} \text { and } \eta_{1} \leq \eta_{2} \leq \cdots \leq \eta_{n}
$$

then every eigenvalue $\lambda$ of $X+i Y$ is contained in the rectangle

$$
\xi_{1} \leq R \lambda \leq \xi_{n}, \eta_{1} \leq d \lambda \leq \eta_{n}
$$

What is the exact range of $\lambda$, for given $\xi_{\nu}$ and $\eta_{\nu}$ ? We shall solve the following slightly more general problem, referring to normal instead of hermitian matrices. Let $\alpha_{1}, \cdots, \alpha_{n}, \beta_{1}, \cdots, \beta_{n}$ be given complex numbers. Describe geometrically the set $\Lambda$ of all numbers $\lambda$ which may occur as eigenvalues of $A+B$, where $A$ and $B$ run over all normal $n \times n$ matrices with eigenvalues $\alpha_{1}, \cdots, \alpha_{n}$ and $\beta_{1}, \cdots, \beta_{n}$ respectively.

To state the results concisely let us denote by the terms circular region and hyperbolic region every set of complex numbers $\xi+i \eta$ which may be described, using some real constants $a, b, c, d$, by

$$
a \xi+b \eta+c\left(\xi^{2} \pm \eta^{2}\right)+d \geq 0
$$

where + refers to the circular, - to the hyperbolic case. We denote by $\left\{\alpha_{\nu}\right\}$ and $\left\{\beta_{\nu}\right\}$ the sets whose elements are $\alpha_{1}, \ldots, \alpha_{n}$ and $\beta_{1}, \cdots, \beta_{n}$ respectively. For every two sets $\Gamma, \Delta$ of complex numbers we denote by $\Gamma+\Delta$ the set whose elements are all $\gamma+\delta$, where $\gamma \in \Gamma, \delta \in \Delta$. Our main result is

THEOREM 1. If $\alpha_{1}, \cdots, \alpha_{n}, \beta_{1}, \cdots, \beta_{n}$ are arbitrary complex numbers, then the set $\Lambda$ defined above can be represented as an intersection:

$$
\Lambda=\prod_{\Gamma}\left(\left\{\alpha_{\nu}\right\}+\Gamma\right)
$$

Received April 16, 1954. This paper was prepared under a National Bureau of Standards contract with the American University.

Pacific J. Math. 5 (1955), 633-638 
where $\Gamma$ runs over all circular regions which contain $\left\{\beta_{\nu}\right\}$.

In the special case considered by Bendixson the result may be simplified as follows.

THEOREM 2. If $\alpha_{1}, \cdots, \alpha_{n}$ are real and $\beta_{1}, \cdots, \beta_{n}$ purely imaginary, then

$$
\Lambda=\cap \Delta
$$

where $\Delta$ runs over all hyperbolic regions which contain $\left\{\alpha_{\nu}\right\}+\left\{\beta_{\nu}\right\}$.

2. Proofs. We recall the following theorem [3, Theorem 2]: Let $\lambda_{1}, \cdots, \lambda_{n}$ be complex numbers, $y$ and $z$ complex $n \times 1$ matrices, $y^{*} y=1$. Denote by $M(y, z)$ the point in real 3-space with rectangular coordinates $\left[R y^{*} z, d y^{*} z\right.$, $\left.z^{*} z\right]$ and by $P(\zeta)$, for every complex number $\zeta$, the point $\left[R \zeta, d \zeta,|\zeta|^{2}\right]$. If, and only if, the convex closure of the n points $P\left(\lambda_{\nu}\right)$ contains $M(y, z)$, then there exists a normal $n \times n$ matrix $L$ with eigenvalues $\lambda_{1}, \cdots, \lambda_{n}$ such that $L y=z$.

Using the notations introduced in $\S 1$ we prove for arbitrary $\alpha_{\nu}, \beta_{\nu}$ :

LEMMA 1. Let $\zeta$ be a complex number. Then $\zeta \in \Lambda$ if, and only if, the convex closure $C$ of $P\left(\alpha_{1}\right), \ldots, P\left(\alpha_{n}\right)$ has a point in common with the convex closure $C^{\prime}$ of $P\left(\zeta-\beta_{1}\right), \cdots, P\left(\zeta-\beta_{n}\right)$.

Proof. (a) Let $\zeta \in \Lambda$. Then there are rormal matrices $A, B$ with spectra $\alpha_{1}, \cdots, \alpha_{n}$ and $\beta_{1}, \cdots, \beta_{n}$ such that

$$
(A+B-\zeta I) y=0
$$

for some normalized vector $y$. Putting

$$
z=A y=(\zeta I-B) y
$$

we conclude from the necessity part of the theorem quoted above that $M(y, z)$ is a common point of $C$ and $C^{\prime}$.

(b) On the other hand, let there be some point

$$
N=\left[\nu_{1}, \nu_{2}, \nu_{3}\right] \in C \cap C^{\prime} \text {. }
$$

Then $v_{1}^{2}+\nu_{2}^{2} \leq \nu_{3}$, hence we have $N=M(y, z)$ for some vectors $y, z$ such 
that $y^{*} y=1$. By the sufficiency part of the theorem quoted above there are normal matrices $A, \widetilde{B}$ with eigenvalues $\alpha_{\nu}, \widetilde{\beta_{\nu}}=\zeta-\beta_{\nu}$ such that $A y=z=\widetilde{B} y$. Define $B=\zeta I-\widetilde{B}$. Then $B$ has the eigenvalues $\beta_{\nu}$ and satisfies $A y=(\zeta I-B) y$, hence $\zeta$ is an eigenvalue of $A+B$ (with $y$ as a corresponding eigenvector).

We transform the "three-dimensional" Lemma 1 into a "two-dimensional" form.

Lemma 2. Let $\zeta$ be a complex number. Then $\zeta \notin \Lambda$ if, and only if, there exists a circle or a straight line separating $\alpha_{1}, \cdots, \alpha_{n}$ from $\zeta-\beta_{1}, \cdots, \zeta-\beta_{n}$.

Proof. From Lemma 1 we know that $\zeta \notin \Lambda$ if, and only if, there exists a plane separating $C$ from $C^{\prime}$, that is, separating

$$
P\left(\alpha_{1}\right), \ldots, P\left(\alpha_{n}\right)
$$

from

$$
P\left(\zeta-\beta_{1}\right), \cdots, P\left(\zeta-\beta_{n}\right)
$$

This means that $\zeta \notin \Lambda$ if, and only if, there are real constants $a, b, c, d$ such that for $\nu=1, \cdots, n$

$$
\begin{gathered}
a R \alpha_{\nu}+b d \alpha_{\nu}+c\left|\alpha_{\nu}\right|^{2}+d>0, \\
a R\left(\zeta-\beta_{\nu}\right)+b d\left(\zeta-\beta_{\nu}\right)+c\left|\zeta-\beta_{\nu}\right|^{2}+d<0 .
\end{gathered}
$$

This proves Lemma 2. We turn to the proof of Theorem 1.

(a) Let $\zeta \in \Lambda$, and let $\Gamma$ be any circular region containing $\beta_{1}, \ldots, \beta_{n}$. We have to show that $\zeta \in\left\{\alpha_{\nu}\right\}+\Gamma$. Now $\zeta-\Gamma$ is a circular region containing $\zeta-\beta_{1}, \cdots, \zeta-\beta_{n}$. By Lemma 2 there is at least one $\alpha_{\rho}$ such that

$$
\alpha_{\rho} \in \zeta-\Gamma, \zeta \in \alpha_{\rho}+\Gamma \subseteq\left\{\alpha_{\nu}\right\}+\Gamma
$$

(b) Let $\zeta \notin \Lambda$. Then Theorem 1 claims that there is a circular region $\Gamma$ containing $\left\{\beta_{\nu}\right\}$ such that $\zeta \notin\left\{\alpha_{\nu}\right\}+\Gamma$. Indeed, interchanging $A$ and $B$ in Lemma 2 we see that there is a circular region $\Gamma$ containing $\beta_{1}, \ldots, \beta_{n}$, but none of the $\zeta-\alpha_{\nu}$.

Theorem 1 being proved, we turn to Bendixson's case where $\alpha_{\nu}=\xi_{\nu}$ is real, and $\beta_{\nu}=i \eta_{\nu}$ with $\eta_{\nu}$ real. We have to show, for any complex number $\zeta$, 
that $\zeta \notin \Lambda$ if, and only if, there is a hyperbolic region which contains all points $\xi_{\mu}+i \eta_{\nu}$, but does not contain $\zeta$. Since this statement obviously is not affected by a translation applied simultaneously to the $\xi_{\mu}+i \eta_{\nu}$ and to $\zeta$, we may assume without loss of generality that $\zeta=0$.

From (2) we know that $0 \notin \Lambda$ if, and only if, there are real numbers $a, b, c, d$ such that

$$
\begin{aligned}
a \xi_{\nu}+c \xi_{\nu}^{2}+d>0 & \\
-b \eta_{\nu}+c \eta_{\nu}^{2}+d>0 & (\nu=1, \cdots, n) .
\end{aligned}
$$

This condition is equivalent to the existence of real numbers, $a, b, c$ such that

$$
a \xi_{\mu}+b \eta_{\nu}+c\left(\xi_{\mu}^{2}-\eta_{\nu}^{2}\right)>0 \quad(\mu, \nu=1, \cdots, n)
$$

This inequality is equivalent to the existence of a hyperbolic region containing all points $\xi_{\mu}+i \eta_{\nu}$, but not 0 .

3. Remarks. (a) It is seen from Theorem 1 that in the determination of $\Lambda$ only the distinct $\alpha_{\nu}$ and the distinct $\beta_{\nu}$ matter. Multiplicities are of no importance.

(b) If $\left\{\tilde{\alpha}_{\rho}\right\} \subseteq\left\{\alpha_{\nu}\right\}$ and $\left\{\tilde{\beta}_{\rho}\right\} \subseteq\left\{\beta_{\nu}\right\}$, then $\tilde{\Lambda} \subseteq \Lambda$.

Proof. For every $\tilde{\sim} \in \widetilde{\Lambda}$ there are normal $r \times r$ matrices $A$ and $B$ with eigenvalues $\widetilde{\alpha}_{\rho}$ and $\widetilde{\beta}_{\rho}$ such that $\lambda$ is an eigenvalue of $A+B$. Define

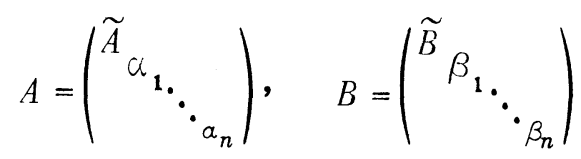

Then $\tilde{\lambda}$ is an eigenvalue of $A+B$. The eigenvalues of $A$ and $B$ coincide with $\alpha_{1}, \cdots, \alpha_{n}$ and $\beta_{1}, \cdots, \beta_{n}$ except for the multiplicities; hence $\tilde{\lambda} \in \Lambda$ by (a).

(c) $\Lambda$ is a closed bounded set, since the sets $\left\{\alpha_{\nu}\right\}+\Gamma$ are closed and there is a bounded circular region $\Gamma$ containing $\left\{\beta_{\nu}\right\}$.

(d) In Bendixson's case every connected component of $\Lambda$ is simply connected.

Proof. By Theorem 2 every point of the complement $\Lambda^{*}$ of $\Lambda$ is the end 
point of some half line which is entirely contained in $\Lambda^{*}$. Moreover by (c), $\Lambda^{*}$ contains the exterior of some circle. Hence $\Lambda^{*}$ is connected.

(e) If $n=2$ in Bendixson's case then Theorem 2 implies that $\Lambda$ is the intersection of Bendixson's rectangle with the rectangular hyperbola passing through the vertices of that rectangle. This result has been previously obtained by W. V. Parker [2, Theorem 1$]$.

(f) The foregoing remarks lead to a simple procedure for constructing $\Lambda$ in Bendixson's case. Let (with a slight change of notation) $\xi_{1}<\xi_{2}<\ldots$ $<\xi_{m}$ be the distinct eigenvalues of the hermitian $n \times n$ matrix $X$, and let $\eta_{1}<\eta_{2}<\cdots<\eta_{k}$ be the distinct eigenvalues of the hermitian $n \times n$ matrix $Y$. We define $\Xi_{\mu}(\mu=1, \cdots, m-1)$ to be that part of the rectangular hyperbola which passes through.

$$
\xi_{\mu}+i \eta_{1}, \quad \xi_{\mu}+i \eta_{K}, \quad \xi_{\mu+1}+i \eta_{1}, \quad \xi_{\mu+1}+i \eta_{K}
$$

which lies in the rectangle with these vertices. Similarly we define $\mathrm{H}_{K}(\kappa=$ $1, \cdots, k-1)$ interchanging the role of the $\xi$ 's and the $\eta$ 's. Then (b) and (e) show that $\Xi_{\mu} \subseteq \Lambda$ and $\mathrm{II}_{K} \subseteq \Lambda$. It is easily seen that the union of all $\Xi_{\mu}$ 's and $\mathrm{H}_{K}$ 's consists of one or more closed Jordan curves each of which is contained in the closed exterior of every other curve, and that no point exterior to all curves belongs to $\Lambda$. On the other hand, the interior of every curve is contained in $\Lambda$, by ( $\mathrm{d})$. Hence $\lambda$ is the largest bounded region whose boundary is the union of $\Xi_{1}, \cdots, \Xi_{m-1}, \mathrm{H}_{1}, \cdots, \mathrm{H}_{K-1}$.

As an example we construct the range $\Lambda$ of the eigenvalues of $X+i Y$ where $X$ and $Y$ are hermitian matrices whose eigenvalues are $0,1,4,8$ and $0,2,3$ (with arbitrary positive multiplicities).

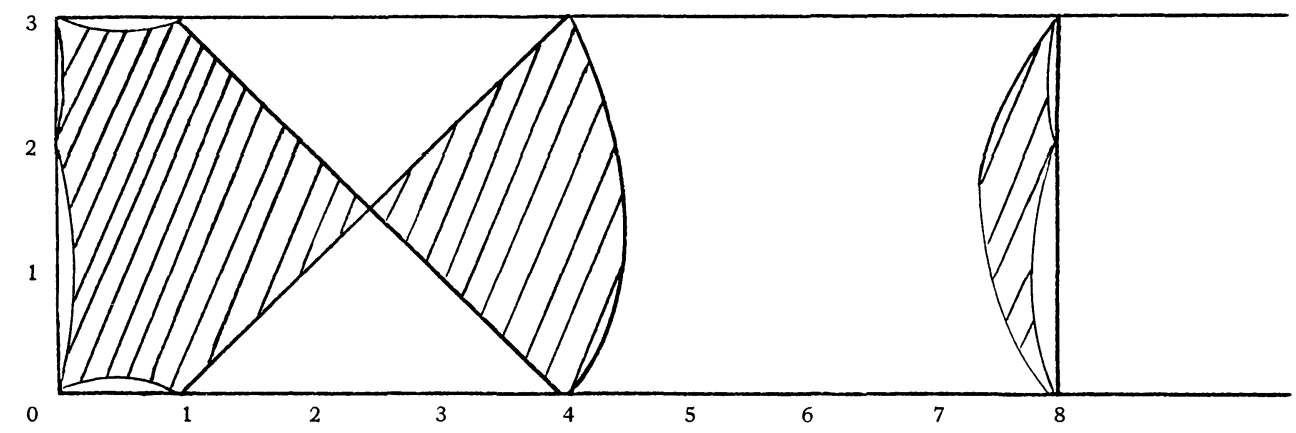




\section{REFERENCES}

1. I. Bendixson, Sur les racines d'une équation fondamentale, Acta Math. 25 (1902), $359-365$.

2. W. V. Parker, Characteristic roots of matrices, Amer. Math. Monthly 60 (1953), $247-250$.

3. H. Wielandt, Die Einschliessung der Eigenwerte normaler Watrizen, Math. Ann. 121 (1949), $234-241$.

AMERICAN UNIVERSITY

UNIVERSITY OF TÜBINGEN

\section{NOTICE}

Supplement I will begin with page 641 . 

